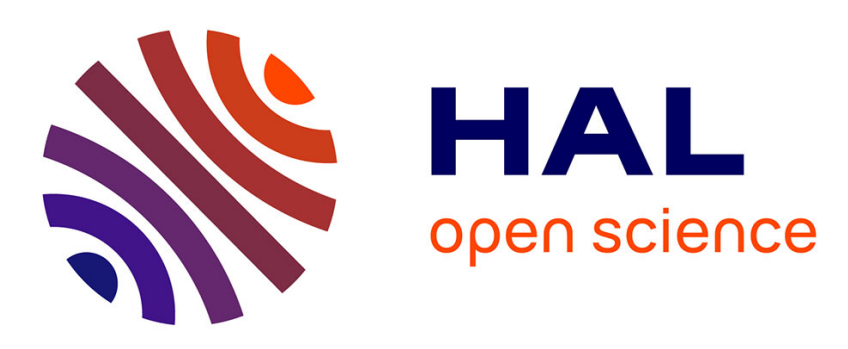

\title{
Material model calibration from planar tension tests on porcine linea alba
}

\author{
Víctor Acostasantamaría, Olivier Siret, Pierre Badel, Gaëtan Guerin, Vít \\ Novacek, Frédéric Turquier, Stéphane Avril
}

\section{To cite this version:}

Víctor Acostasantamaría, Olivier Siret, Pierre Badel, Gaëtan Guerin, Vít Novacek, et al.. Material model calibration from planar tension tests on porcine linea alba. Journal of the mechanical behavior of biomedical materials, 2015, 43, pp.26-34. emse-01108503

\section{HAL Id: emse-01108503 \\ https://hal-emse.ccsd.cnrs.fr/emse-01108503}

Submitted on 23 Jan 2015

HAL is a multi-disciplinary open access archive for the deposit and dissemination of scientific research documents, whether they are published or not. The documents may come from teaching and research institutions in France or abroad, or from public or private research centers.
L'archive ouverte pluridisciplinaire HAL, est destinée au dépôt et à la diffusion de documents scientifiques de niveau recherche, publiés ou non, émanant des établissements d'enseignement et de recherche français ou étrangers, des laboratoires publics ou privés. 


\title{
Material model calibration from planar tension tests onporcine linea alba.
}

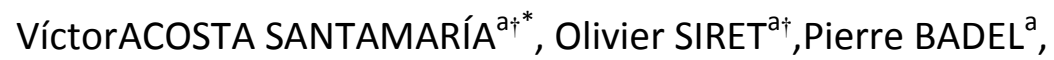 \\ Gaëtan GUERIN ${ }^{b}$, Vít NOVACEK ${ }^{b}$ Frédéric TURQUIER ${ }^{b}$, Stéphane AVRIL ${ }^{a}$ \\ ${ }^{a}$ Ecole Nationale Supérieure des Mines de Saint Etienne, CIS-EMSE, CNRS:UMR5307, \\ LGF, 158 cours Fauriel, 42023 Saint-Etienne Cedex 2, France \\ ${ }^{\mathrm{b}}$ Covidien Surgical Solutions, Sofradim Production, 116 Avenue du Formans, 01600 \\ Trévoux, France \\ *Corresponding author:victor.acosta@emse.fr, \\ Tel: +33.4.77.42.01.88, Fax:+33.4.77.49.96.94 \\ ${ }^{\dagger}$ These authors contributed equally to this manuscript.
}

\begin{abstract}
The purpose of this study was to determine biomechanical properties of linea alba subjected to transverse planar tension and to compare its behavior at different locations of the abdominal wall. Samples of linea albafrom five different porcine abdominal walls were tested in planar tension. During these tests, strain maps were measured using thestereodigital image correlation (S-DIC) technique. The strain maps were used to derive the properties of differenthyperelastic material models. It was shown thatthe Ogden model and the Holzapfel-Gasser-Ogdenmodel areappropriate to reproduce the response in planar tension. The linea alba located above the umbilicus was significantly more compliant than below the umbilicus. This differencewhich is reported for the first time here is consistent with the tissuemicrostructureand it is discussed within the perspective of clinically-relevant numerical simulations.
\end{abstract}

Keywords:abdominal wall; linea alba; S-DIC;mechanical parameters identification; hyperelastic models.

\section{Introduction}

Abdominal wall (AW) is a complex musculo-aponeurotic structure made of 2 symmetrical parts that join in the sagittal plane along a mid-line named linea alba, as explained byPodwojewski et al., 2013 and López-Cano et al., 2013. It is the most common site for laparotomy incision (World Health Organization, 2003) and it plays a key role in AW biomechanics during and after closure of incisions or hernia defects (van't Riet et al., 2002).From an anatomic point of view, linea alba is a midline raphe formed by interweaving of anterolateral abdominal muscle aponeuroses, as described by Gray, 1918 and Dyce et al., 2009 for respectively human and porcine anatomy. Porcine tissue have a similarly sized heart and body length to humans but it is unknown if they share histological similarities (Cooney et al., 2014). As commonly described, the width of linea albavaries significantlyfrom cranial to 
caudal. Typical porcine linea alba was observed to have an average width of $20 \pm 5$ $\mathrm{mm}$ (Cooney et al., 2014).

The mechanical properties oflinea albawerecharacterizedby different authors: (Rath et al., 1996), (Grässel et al., 2005), (Förstemann et al., 2011), (Martins et al., 2012), (Podwojewski et al., 2013), (Tran et al., 2014) and (Cooney et al., 2014). These authorscharacterizedlinear elastic or hyperelastic properties to model the AW biomechanical behavior. Cooney et al., 2014 applied uniaxial and equibiaxial tension tests to identify the mechanical properties of the tissue.

The main goal of the present study is to characterize the mechanical behavior of the linea alba in the situation of a laparotomy closure. Atensile test, with $100 \%$ of the deformation in the transverse direction, is applied to obtain the mechanical properties. This test was chosen because it is globally similar to the loadingapplied to the linea alba during a laparotomy closure by suture.

Additionally, experimental studiesmay yield material properties that can be input in finite element models, as performed for instance by Hernández-Gascón et al., 2013 and Cooney et al., 2014.Finite-element model of midline laparotomy closure requiring knowledge of such material properties is currently under development (Nováček and Turquier, 2013). An accurate characterization of the biomechanical properties of linea alba is therefore an essential issue for the validity of the models.

In this work, we present anin vitro mechanical characterization oflinea alba using theplanar tension tests. The purpose is to compare the biomechanical properties at different locations of the abdominal wall (supraumbilical and infraumbilical zones). Samples of linea albawere harvested from five porcine abdominal walls. Full-field deformation measurementswereperformed, for the first time ever, by stereo digital image correlation (SDIC).Finally, an inverse approach was used to derive the parameters of different material modelsfrom the response of the tissue to each test.

\section{Materials and methods}

\subsection{Preparation and dimensions of the specimens}

Anterolateral AWs of five female pigs, aged $4-5$ months and weighing about $45 \mathrm{~kg}$, were used for the current study. The AWs were removed from the animals less than 30 min after euthanasia at the VetAgro Sup - Veterinary Campus of Lyon (Marcy l'Etoile, France), and then kept frozen at $-20{ }^{\circ} \mathrm{C}$ until testing. The AWs were cut along xiphoid process and costal margins and along pubic bones and iliac crests. Lateral incisions were done between iliac spines and the lower part of the rib cage. All the layers were preserved: muscles, aponeuroses, adipose tissue, skin and peritoneum. In a second phase, AWswere thawed at room temperature $16 \mathrm{~h}$ in order to cut outsamples.

After dissecting skin and adipose tissue on the ventral side and peritoneum on the dorsal side, 80 to $100 \mathrm{~mm}$ long rectangular samples were cut for the tensile tests. Thirty samples of linea alba were obtained from five different porcine abdominal walls. The linea alba tissue was divided in the supraumbilical and infraumbilical zones (above and below umbilicus, respectively). For each abdominal wall six samples were considered. Additionally, for each 
zone three different samples were obtained, see Figure $1 \mathrm{a}$. With respect to the umbilicus, samples 1 and 6 are the most distant and samples 3 and 4 are the closest, see Figure $1 \mathrm{a}$. Before the test, the samples dimensions were measured with a digital caliper.Considering the size of the samples and the relative flatnessof the surface, caliper seems to be an adequate technique of thickness measurement (O'Learyet al., 2013; Leeet al., 1996).

The sample height is denoted $H_{0}$. For the supraumbilical and infraumbilical zones the reported $H_{0}$ was $24.5 \pm 5.37 \mathrm{~mm}$. Only the middle part of the sample is linea alba tissue, the remainder of the sample is composed of rectus abdominis muscle (RM) and rectus sheaths (RS), see Figure $1 \mathrm{~b}$. The width and the thickness of the linea alba are denoted $W_{0}$ and $t_{0}$ respectively, see Figure 1b.

\subsection{Multiphoton imaging}

Before the mechanical tests, collagen and elastin fibers were imaged using two photon excitation microscopy in a $500 \times 500 \times 150 \mu \mathrm{m}^{3}$ volume $(150 \mu \mathrm{m}$ being the depth) located inthe middle part of the samples (linea alba).The confocal microscope was Nikon A1R MP PLUS ${ }^{\circledR}$ (ivtv.ec-lyon.fr). The tissue was excited with an 800nm laser. The second harmonic generation (SHG) signal of the collagen fibers was collected through a 380-420 nm bandpass filter using a 20x objectives. The autofluorescence signal was also collected and subtracted to the SHG signal in order to image the elastin fibers.

\subsection{Planar tension tests}

During the experimental tensile test, only the middle tissue (i.e. linea alba), was subjected to tension. The $R M$ and $R S$ tissues were pressed by hydraulic clamps with 3 bars of pressure. Consequently, no sliding of the specimens was reported.In the geometrical definition of the samples, $H_{0}$ is larger than $W_{0}$. The assumption of planar tension was satisfied in the experimental protocol (the height remained constant during the tensile test). In the tensile test the axial direction corresponded to the width (direction 2 in the global coordinate system, see Figure 1c). The transversal direction corresponded to the height of the specimen (direction 1). Finally, the out-of-plane dimension corresponded to the thickness (direction 3).Only the transverse direction was characterized with this experimental protocol.

The planar tension test was performed in an Instron machine (3343 - Series 3300 Load Frames). The testing machine was instrumented with a load cell (2519 Instron Series) to measure the axial force resulting of an imposed displacement, see Figure 1c. The vertical displacement was imposed to the movable clamp while the bottom clamp remained static, see Figure 1c. The test was performed at $0.2 \mathrm{~mm} / \mathrm{s}$ cross-head velocity. The test lasted less than 10 minutes ( $7 \leftrightarrow 10 \mathrm{~min}$ ). No significant dehydration effects were observed during the tests (Martins et al., 2011).

\subsection{Measurement of strain maps by 3D-S-DIC}

A non-contacting full-field measurement technique,called Stereo-Digital Image Correlation (S-DIC), was used tomeasure local displacements in the soft tissue (Sutton et al., 2009). The S-DIC system (GOM, 5M LT) was composed of two 8-bit CCD cameras equipped with $50 \mathrm{~mm}$ lenses with a resolution of $1624 \times 1236$ pixels. Even for tensile tests a single camera is very sensitive to parasitic out of plane motions, even tiny, whereas a stereo system (with two cameras) is immune to this problem (Avril et al. 2008). A random speckle pattern was 
carefully deposited on the surface of each specimenusingspray paint as shown infigure 1c. Previous studies have found the thin layer of spray paint has a negligible effect on the mechanical properties (Zhang et al. 2002).

During the mechanical tests, pictures were taken with both cameras of the S-DIC system at a frequency of $5 \mathrm{~Hz}$. After the test was completed, the collected images were analyzed using the commercial correlation software ARAMIS (GOM, v. 6.2.0) to determine the three dimensional displacement of the tissue surface. For the image analysis, a facet size of $21 \mathrm{px}$ and a facet step of $10 \mathrm{px}$ were chosen based on the speckle pattern dot size, distribution, and contrast. A finite difference method was used to calculate the engineering strain locally in the specimen (Avril et al., 2010).

\subsection{Material models}

Hyperelastic models are commonly used to describe the mechanical behavior of rubber-like materials and soft tissues (Ogden, 1997). These constitutive models are definedusinga strain energy density function $W(\boldsymbol{F})$ where $\boldsymbol{F}$ is the deformation gradient tensor.

In the present study, it was chosen to consider the three following strain energy density functions: Neo-Hookean, Ogden and Holzapfel-Gasser-Ogden (Holzapfel et al., 2000).

Moreover, it was assumed that the material remained incompressible, i.e. $\operatorname{det}(\boldsymbol{F})=1$. Consequently, in terms of principal stretch $\lambda_{i}$, it may be written: $\lambda_{3}=\left(\lambda_{1} \lambda_{2}\right)^{-1}$.

The Neo-Hookean strain energy density function is written such as:

$$
W\left(\lambda_{1}, \lambda_{2}, \lambda_{3}\right)=\mathrm{C}_{10}\left(\lambda_{1}^{2}+\lambda_{2}^{2}+\lambda_{3}^{2}-3\right)
$$

where $C_{10}$ is the unique parameter to be identified.

The strain energy density function of the Ogden model(Ogden et al., 2004)is expressed in terms of the principal stretches such as:

$$
W\left(\lambda_{1}, \lambda_{2}, \lambda_{3}\right)=\frac{2 \mu}{\alpha^{2}}\left(\lambda_{1}^{\alpha}+\lambda_{2}^{\alpha}+\lambda_{3}^{\alpha}-3\right)
$$

where $\mu$ and $\alpha$ are two parameters to be identified.

A simplified form of theHolzapfel-Gasser-Ogden model(Holzapfel et al., 2000)was used here. The Neo-hookean term existing in the original model was neglected. Moreover, given the collagen fiber architecture described by (Axer et al., 2001), it was assumedthat the stiffness is initially twice as large in the transverse direction of linea albaas in the axial direction, which leads to an initial fiber angle of $35.26^{\circ}$. Hence, the strain energy density function was expressed in terms of the principal stretches such as:

$$
W\left(\lambda_{1}, \lambda_{2}, \lambda_{3}\right)=\frac{k_{1}}{2 k_{2}}\left\{\exp \left[k_{2} / 9\left(2 \lambda_{1}^{2}+\lambda_{2}^{2}\right)^{2}\right]-1\right\}
$$

where $k_{1}$ and $k_{2}$ are two material parameters to be identified. 


\subsection{Material model calibration in planar tension tests}

The strain data obtained in planar tension tests were averaged out across the area of linea alba. This confirmed that the shear strain could be neglected becausewe had $\lambda_{1} \approx 1$, where direction 1 was perpendicular to the tension direction (Figure 2). Then the main principal stretch value should be the direction of tension. It wasdenoted: $\lambda_{2}:=\lambda$.

The load value, denoted $F$, could be related to $\lambda$ and to the models of Eq. 1,2 or 3 such as:

$$
F=2 t_{0} H_{0}\left(\lambda^{2} \partial W / \partial \lambda_{2}^{2}-\lambda^{-2} \partial W / \partial \lambda_{3}^{2}\right) \lambda^{-1}
$$

In order to determine the parameters of Eq. 1, 2 or 3 predicting load values that maximize the agreement with the measured load values, thefollowing objective function was minimized: $\theta(\beta)=\sum\left(F_{i}-\hat{F}_{i}(\beta)\right)^{2}$ where $F_{i}$ are the experimental load values and $\left(\hat{F}_{i}(\beta)\right.$ are the predictedload values for a set of model parameters $\beta$. Index $i$ represents the step number at which the measurement was performed. Given a random initialization point and convergence criteria, the optimal model parametersset $\beta^{*}$ were obtained.The coefficient of determination $R^{2}=1-R S S / T S S$ was evaluated, where $R S S=\sum\left(F_{i}-\hat{F}_{i}\right)^{2}$ and $T S S=$ $\sum\left(F_{i}-\bar{F}\right)^{2}$ where $\bar{F}$ is the mean value of the experimental loads measured throughout a given test. Optimization was performed using the function Lsqcurvefit in the Optimization Toolbox of MatLab ${ }^{\circledR}$ which solves nonlinear data-fitting problems in a least-squares sense.

\subsection{Statistical analysis}

Two inferences were considered for the statistical analysis in the comparison of the geometrical dimensions $\left(W_{0}\right.$ and $\left.t_{0}\right)$. A normal distribution was tested by Anderson-Darling test for the parametric inference. In this case the test determines whether the data follow a normal distribution for a pre-determined level of significance of $95 \%$ ( $p$-value $\geq 0.05$ ). The results were presented as mean \pm standard error. If the $p$-value was lower than the selected criterion, a non-parametric inference was applied to answer the null hypothesis test value with Wilcoxon test. The results were presented as median \pm confidence intervals. A KruskalWallis test was applied to define the statistical differences between populations. The statistical significance was set at $p$-value $\leq 0.05$ with $95 \%$ confidence interval. The statistical methods applied were calculated using Minitab ${ }^{\circledR}$.

\section{Results}

\subsection{Dimensions of the specimens}

From five different abdominal walls, thirty samples of linea alba were obtained. Two zones with respect to the umbilicus were defined for the abdominal wall (supraumbilical and infraumbilical). Additionally, for each abdominal wall six samples were considered, three samples for the supraumbilical zone and three for the infraumbilical zone.A statistic analysis was applied to evaluate the geometrical dimensions of the samples for both zones (supraumbilical and infraumbilical). The objective wasto determine the significant differences for the width $\left(W_{0}\right)$ and thickness $\left(t_{0}\right)$ of the experimental samples. For the first approach,we considered a descriptive statistics formulation assuming a parametric inference, normal distribution of the data and a non-random sampling. 
For the supraumbilical and infraumbilical zones the reported data for $W_{0}$ and $t_{0}$ showed a non-normal distribution ( $p$-value $<0.05$ ). With this result, it was not possible to demonstrate that the samples represent the same population. Therefore, it was necessary to consider a non-parametric inference. With this approach the populations were compared with the corresponding median and their respective confidence intervals, see Figure 2.

Regarding the supraumbilical zone, an increase in the distance with respect to the umbilicus corresponded to a decreasedof $W_{0}$, see Figure $2 a$, whereas $t_{0}$ showed an opposite tendency, see Figure $2 \mathrm{~b}$. For $W_{0}$ and $t_{0}$ the statistical treatment determined significant differences between the samples 1 and 3 ( $p$-value $<0.05$ ), defining them as non comparable samples (see Figure 2). Regarding the infraumbilical zone, an increase in the distance with respect to the umbilicus corresponded to a decrease in $W_{0}$ and $t_{0}$, see Figure 2. However, the statistical treatment reported significant differences only for $W_{0}$ and between samples 4 and 6 ( $p$ value $<0.05)$, see Figure $2 a$. Additionally, comparing the geometrical dimensions $W_{0}$ and $t_{0}$ for the supraumbilical and infraumbilical samples closer to the umbilicus ( 3 and 4 , respectively), the non-parametric inference reported non comparable samples ( $p$-value $<0.05)$, see Figure 2 . Therefore, the samples near the umbilicus could not be compared between them, nor with samples 1 and 6 of the supraumbilical and infraumbilical regions, respectively. Finally, the analysis determined statistical differences ( $p$-value $<0.05$ ) between the supraumbilical and infraumbilical samples $(1-2)$ and $(5-6)$, respectively (see Figure 2 ).

Considering the above comparative results for the infraumbilical and supraumbilical zones, the samples closer to the umbilicus reported differences with the remaining samples. In conclusion, considering the geometrical factors $\left(W_{0}\right.$ and $\left.t_{0}\right)$, samples 3 and 4 could not be taken into account to identify the parameters of the mechanical behavior of the linea alba. The final geometrical dimensions are reported in Table 1. With respect to the umbilicus distance, $W_{0}$ was reduced by $36 \%$ between samples 2 and 1 in the supraumbilical zone and $t_{0}$ was increased by $25.45 \%$. Moreover, for the infraumbilical region, $t_{0}$ was almost constant and $W_{0}$ was reduced by $16.39 \%$ between samples 5 and 6 , with respect to the umbilicus distance.

\subsection{Microscopic observations}

As linea alba is prolongation of rectus sheaths and, more generally, fascias of lateral muscles, its structure is known to follow fiber orientation of lateral abdominal muscles. Observations using the second harmonic generation confocal microscope revealed differences between collagen fiber orientations on the outer (ventral) side of the samples and on the inner (dorsal) side of the samples. Transverse fiber orientations were predominant on the dorsal side for both supraumbilical and infraumbilical samples whereas oblique fiber orientations were predominant on the ventral side in supraumbilical samples only, see Figure 3 . Our findings are in good agreement with (Axer et al., 2001) except for ventral side in infraumbilical samples which may be explained by different anatomy of pigs compared to humans as pointed out by (Cooney et al, 2014) especially when considering differences in muscle insertions.

\subsection{Planar tension tests}


All the tissue samples displayed a similar mechanical response under planar tension, see Figure 4. Three stages are distinguishable in a typical stress-strain curve: an initial stage from which the hyperelastic parameters can be identified (shown with a solid line in Figure 4), then a tearing stage with an inversion of the curvature. The laststage represents the rupture where the stress returns to zero. The tearing and rupture always occurred in the muscular bits of the samples that were pressed with the clamps. The strain levels reported for the linea alba were relatively low (less than 10\%), sufficiently below the damage threshold for this tissue. In general, the highest stress levels were reported for the infraumbilical samples, whereas the supraumbilical samples showed the highest strain levels, see Figure 4.

The first stage of these curves was used to deduce the hyperelastic parameters. In order to validate the planar tension hypothesis, the variation of the width of the specimen was determined thanks to the displacements provided by the S-DIC technique. The average transverse strain remained less than $0.1 \%$, which validates the assumption of planar tension tests $\left(\lambda_{1} \approx 1\right)$.

Ten specimens were rejected after the statistical analysis that was applied to evaluate the geometrical dimensions of the samples. These specimens correspond to the samples closer to the umbilicus (samples 3 and 4), see Figure 1. To define the hyperelastic parameters weconsidered twenty samples of linea alba from five different abdominal walls. For each abdominal wall four specimens were considered, two samples for the supraumbilical zone (1 and 2) and two for the infraumbilical zone (5 and 6), see Figure 1 . For each region, the identified parameters reported a normal distribution ( $p$-value $>0.05)$, assuming a descriptive statistics formulation. However, one and two samples for the infraumbilical and supraumbilical zones respectively, reported mechanical parameters that were considered like outliers.The identified hyperelastic parameters are reported in Table 2, for the three evaluated models: Neo-Hookean, Ogden and Holzapfel-Gasser-Ogden (Eq. 1, 2 and 3, respectively). The data were reported with the corresponding mean \pm standard deviation.

Comparing the mechanical parameters between the supraumbilical and infraumbilical zones thestatistical treatment determined significant differences.Regarding the Neo Hookean model and the parameter $\mathrm{C}_{10}$, for both zones significant differences was determined ( $\mathrm{p}$-value $<0.05$ ), defining them as non comparable parameters. Moreover, the $C_{10}$ parameters reported for each zone differ in $85 \%$.Concerning the Ogden model, the statistical treatment reported significant differences only for $\mu$ parameter ( $p$-value $<0.05$ ). The $\mu$ parameters reported for the infraumbilical and supraumbilical zones differ in $92 \%$. For the alpha parameters and between the regions, the difference is $7.95 \%$.Finally, for the HolzapfelGasser-Ogden model the significant differences was reported only for $k_{1}$ parameter ( $p$-value $<0.05)$. For the supraumbilical and infraumbilical zones the $k_{1}$ and $k_{2}$ parameters differsin 91 and $18 \%$, respectively.

The Ogden and Holzapfel-Gasser-Ogden models provided excellent fits with determination coefficients of 0.99 and 1.0 for the supraumbilical and infraumbilical samples, respectively, see Table 2. The Neo-Hookean model seemed less appropriate with determination coefficients of 0.73 and 0.89 , respectively. This may be explained by the almost systematic Jshaped stress-strain curves. In this sense, the parameters of Ogden and Holzapfel-GasserOgden models were appropriate to reproduce the J-shape. 
Using the identified parameters with the Holzapfel-Gasser-Ogden model, the stress-stretch curves in planar tension for the supraumbilical and infraumbilical samples were plotted on the same graph, see Figure 5. Twenty samples of linea alba were collected for the study, ten for each zone (supraumbilical and infraumbilical). The results are presented as mean \pm standard deviation for different stress and strains levels.

For the supraumbilical zone, an increase in the stretch increases the dispersion with respect to the stress mean values that are reported. Moreover, the infraumbilical region shows a similar tendency with a higher average deviation compared with the results of the supraumbilical zone, see Figure 5. Finally, an increase in the stress levels for the supraumbilical and infraumbilical samples, increases the dispersion with respect to the stretch mean values and with similar trends, see Figure 5.

Considering the previous results of the geometrical dimensions comparison, the analyses determined statistical differences between the supraumbilical and infraumbilical zones. Additionally, the planar tension results reported differences in the stress and strain levels for both zones. This explains the differences for the mechanical behavior and parameters found for the linea alba.

\section{Discussion}

The purpose of this study was to determine the biomechanical properties of linea alba subjected to planar tension (representing physiological healthy conditions). This is the first study ever reporting simultaneously the biomechanical response of linea alba to planar tension both at infraumbilical and supraumbilical location. Indeed, results reported in the literature focused on infraumbilical samples from which tensile specimens could be more easily cut. As the supraumbilical linea alba is narrower and thicker, this does not permit to cut classical tensile samples. In our study this drawback was overcome. The linea alba and the surrounding muscles were tested in a single tensile test. The non-contact S-DIC technique was used to measure the strain fields in the linea alba region.

Even if the current study was performed on porcine tissues, the distribution patterns were similar to human ones, as was observed by (Axer et al., 2001). We found significant differences between supraumbilical and infraumbilical samples, the latter being significantly stiffer. Such a difference was not reported by (Grässel et al., 2005) for the reason explained above (impossible to carry out a traditional tensile test protocol on supraumbilical linea $a l b a)$. However, structural and microstructural observations confirmed the differences between supraumbilical and infraumbilical samples. Indeed, supraumbilical linea alba is thicker and narrower than infraumbilical linea alba because it contains a layer of collagen fibers diagonally oriented. This structural difference between supraumbilical samples and infraumbilical linea alba was reported by (Axer et al. 2001). The presence of diagonal fibers in supraumbilical linea alba, extending from aponeuroses of oblique muscles, accounts for a more compliant response to transverse planar tension than in infraumbilical linea alba where most of the fibers are oriented in the transverse direction.

Above the umbilicus, transverse deformation of abdominal wall is limited by thoracic cage. Abdominal wall in this region experiences more deformation resulting from trunk movement 
then intra-abdominal pressure. Oblique fibers in linea alba transmit tensions especially in trunk rotation. The abdominal wall, under the umbilicus, has to respond more to a loadinginduced by intra-abdominal pressure. The tensions, in linea alba, are transmitted mostly by transverse fibers. Our findings confirm such hypothesis: linea alba function correlates well with microstructure which determines macroscopic mechanical behavior. However, this study was done on porcine tissues and validity of this hypothesis would need to be confirmedwith respect to human anatomy.

The stress/stretch curve of linea alba below the umbilicus was very close to that reported by (Cooney et al., 2014) for linea alba tested in the transverse direction, see Figure 6. Generally, the stress experienced by linea alba was of the same order of magnitude as reported by (Abdelounis et al., 2013) for anterior rectus sheath, see Figure 6.

The stress applied in our tests remained below the strength values reported by (Hollinsky \&

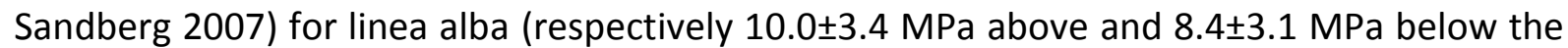
umbilicus). The global behavior of infraumbilical linea alba to planar tension is in very good agreement with the results obtained on human samples by (Grässel et al., 2005), even though these authorsdid not observe significant differences in position (supraumbilical vs. infraumbilical).(Rath, et al., 1997) also reported for human rectus sheath a coefficient of elasticity greater for the infraumbilical than for the supraumbilical tissue, which is the same tendency as our results.

Material models and corresponding parameters are fundamental in development of robust computational models simulating healthy physiological conditions, and also midline laparotomy closure and/or abdominal wall repair in case of hernia. Such models would be helpful in assessment of performance of medical devices and surgical techniques in order to improve the clinical outcome.Finite element models of AW were already published by our group (Guérin and Turquier, 2013) or others(Hernández-Gascón et al., 2013 and Cooney et al., 2014). They need to be fed with material parameters. Most of the finite-element models of soft tissues require hyperelastic models (Holzapfel et al., 2000). Previous data published by (Grässel et al., 2005 and Cooney et al., 2014) provided elastic and hyperelastic parameters of linea alba, respectively. However, our study is the first one to report the significant differences for the material properties of the linea alba between the supraumbilical and infraumbilical zones.

The use of full-field strain measurements becomes more and more commonplace in soft tissue biomechanics (Romo et al., 2014; Brunon et al., 2010). In the current study, it permitted a contactless measurement of strains in planar tension. Usually, in experimental tests carried out on soft tissues, strains are measured by dividing the displacement of the tensile machine by the initial length of the specimen. Here this approach was not possible because the tensile machine induced deformation not only of linea alba but also of the muscular parts surrounding it. Therefore, the total displacement of the tensile machine was the sum of the deformation of all the different tissues constituting the samples. Thanks to full-field strain measurements with the S-DIC technique, it was possible to assess the average strain of linea alba only and eventually to derive the intrinsic material properties of linea alba only. 
This study presents a number of limitations that could be addressed in future studies:

1. The axial stretch applied in planar tension tests remained relatively small (stretch limited to $1.1 \mathrm{max}$ ). This was due to the fact that tearing always occurred in the muscular tissue squeezed in the grips before exceeding $10 \%$ strain in thelinea alba. In a human cadaveric study, (Nováček et al., 2014) observed average anterior abdominal wall strain of $5 \%$ at intra-abdominal pressure of $4 \mathrm{kPa}$. In comparison, (Grässel et al., 2005) applied strains varying from $5 \%$ to more than $30 \%$ by testing linea alba alone. The main asset of our protocol is that the samples of linea alba were not damaged during the planar tension tests, and the same samples can be used for other experimental protocols like the suture pull-out test. No macroscopic damage was observed. The applied strain was lower or of the same order of magnitude as the in vivo strain experienced by abdominal wall (Śmietański et al., 2014; Szymczak et al., 2014).

2. Our studies focused on porcine linea alba. This represents some advantage as pigs are very often used as animal models for investigating laparotomy closure by suture and for surgeon practice. However, the possible differences between porcine models and humans may exist. We noticed a significant difference between supra-umbilical and infra-umbilical responses in planar tension. Such difference was not reported in humans even if the protocol for testing human linea alba was less sophisticated and generated a lot of scatter in the results (Grässel et al., 2005).

3. The effect of freezing and thawing on the biomechanical properties of the samples was not characterized. Moreover, all the tests were carried out at room temperature. Repeating the tests in conditions closer to the physiological ones should be the object of another study.

\section{Conclusions}

Samples of linea albafrom five different porcine abdominal wallswere tested in planar tension. It was shown that the Ogden model and the Holzapfel-Gasser-Ogden models are appropriate to reproduce their mechanical response. The linea alba located above the umbilicus was significantly more compliant than below the umbilicus. This difference which is firstly reported here is consistent with the tissue microstructure. These results have important consequences as they indicate that regional variations of material properties should be considered to set-up finite-element models of AW in healthy physiological conditions and of AW during laparotomy closure by suture. 


\section{References}

Avril, S., Feissel, P., Pierron, F., Villon, P. (2010), 'Comparison of two approaches for controlling the uncertainty in data differentiation: application to full-field measurements in solid mechanics', Measurement Science and Technology 21(1), 15703-15713.

Axer, H., v. Keyserlingk, D. G. \& Prescher, A. (2001), 'Collagen fibers in linea alba and rectus sheaths: I. general scheme and morphological aspects', Journal of Surgical Research 96(1), $127-134$.

Brunon, A., Bruyère-Garnier, K., Coret, M. (2010), 'Mechanical characterization of liver capsule through uniaxial quasi-static tensile tests until failure', Journal of Biomechanics 43, 2221-2227.

Dyce, K. M., Sack, W. O. \& Wensing, C. J. G. (2009), Textbook of Veterinary Anatomy, 4th edn, Elsevier - Health Sciences Division, chapter 36-The Abdomen of the Pig, pp. 776-785.

Förstemann, T., Trzewik, J., Holste, J., Batke, B., Konerding, M., Wolloscheck, T. \& Hartung, C. (2011), 'Forces and deformations of the abdominal wall-a mechanical and geometrical approach to the linea alba', Journal of Biomechanics 44(4), 600 - 606.

Gray, H. (1918), Anatomy of the human body, 20th edn, Philadelphia: Lea \& Febiger.

Grässel, D., Prescher, A., Fitzek, S., v. Keyserlingk, D. G. \& Axer, H. (2005), 'Anisotropy of human linea alba: A biomechanical study', Journal of Surgical Research 124(1), $118-125$.

Guérin, G., Turquier, F. (2013), 'Impact of the defect size, the mesh overlap and the fixation depth on ventral hernia repairs: a combined experimental and numerical approach',Hernia $17(5), 647-655$.

Hernández-Gascón, B., Mena, A., Peña, E., Pascual, G., Bellón, J. \& Calvo, B. (2013), 'Understanding the passive mechanical behavior of the human abdominal wall', Annals of Biomedical Engineering 41(2), 433-444.

Holzapfel, G., Gasser, T. \& Ogden, R. (2000), 'A new constitutive framework for arterial wall mechanics and a comparative study of material models', Journal of Elasticity 61, 1-48.

López-Cano, M., Armengol, M., Quiles, M. T., Biel, A., Velasco, J., Huguet, P., Mestre, A., Delgado, L. M., Gil, F. X. \& Arbós, M. A. (2013), 'Preventive midline laparotomy closure with a new bioabsorbable mesh: An experimental study', Journal of Surgical Research 181(1), 160 169.

Martins, P., Peña, E., Jorge, R. N., Santos, A., Santos, L., Mascarenhas, T. \& Calvo, B. (2012), 'Mechanical characterization and constitutive modelling of the damage process in rectus sheath', Journal of the Mechanical Behavior of Biomedical Materials 8(0), 111-122. 
Nováček, V., Dunn, R., Ignotz, R. \& Turquier, F. (2014) 'A New Methodology for Abdominal Wall Biomechanical Strain Analysis', In: 16th Annual Hernia Repair conference, Las Vegas, USA, 12-15 March, 2014.

Ogden, R.W. (1997), 'Non-linear elastic deformations', Dover Publications.

Ogden, R. W., Saccomandi, G. \& Sgura, I. (2004), 'Fitting hyperelastic models to experimental data', Computational Mechanics 34, 484-502.

Podwojewski, F., Otténio, M., Beillas, P., Guérin, G., Turquier, F. \& Mitton, D. (2013), 'Mechanical response of animal abdominal walls in vitro: Evaluation of the influence of a hernia defect and a repair with a mesh implanted intraperitoneally', Journal of Biomechanics 46(3), $561-566$.

Rath, A., Attali, P., Dumas, J., Goldlust, D., Zhang, J. \& Chevrel, J. (1996), 'The abdominal linea alba: an anatomo-radiologic and biomechanical study', Surgical and Radiologic Anatomy 18(4), 281-288.

Romo, A., Avril, S., Badel, P., Duprey, A., Favre, J.P. (2014), 'In vitro analysis of localized aneurism rupture', Journal of Biomechanics 47(3), 607-616.

Sutton, M., Orteu, J. \& Schreier, H. (2009), Image Correlation for Shape, Motion and Deformation Measurements: Basic Concepts, Theory and Applications, Springer.

van't Riet, M., Steyerberg, E. W., Nellensteyn, J., Bonjer, H. J. \& Jeekel, J. (2002), 'Metaanalysis of techniques for closure of midline abdominal incisions', British Journal of Surgery 89(11), 1350-1356.

World Health Organization (2003), Surgical Care at the District Hospital (SCDH) manual Chap.6 Laparotomy and abdominal trauma.

Zhang D, Eggleton C, Arola D (2002) Evaluating the mechanical behavior of arterial tissue using digital image correlation. Exp Mech 42(4):409-416. 


\section{TABLES}

Table 1. Geometrical dimensions of linea alba samples (width and thickness).

\begin{tabular}{|c|c|c|c|}
\hline \multicolumn{4}{|c|}{ Geometrical Dimensions - Estimated Median [Confidence Interval] } \\
\hline Zone & Samples & Width $W_{0}(\mathrm{~mm})$ & Thickness $\mathrm{t}_{0}(\mathrm{~mm})$ \\
\hline \multirow{2}{*}{ Supraumbilical } & 1 & $3.2[-1.25 ;+2.15]$ & $3.45[-0.7 ;+1.05]$ \\
\hline & 2 & $5.0[-1.55 ;+5.80]$ & $2.75[-0.95 ;+0.9]$ \\
\hline \multirow{2}{*}{ Infraumbilical } & 5 & $15.0[-1.85 ;+3.35]$ & $1.5[-0.4 ;+0.2]$ \\
\hline & 6 & $12.5[-3.25 ;+3.50]$ & $1.5[-0.3 ;+0.15]$ \\
\hline
\end{tabular}


Table 2. Material parameter identification of 3 hyperelastic models for supraumibical and infraumbilical zones.

\begin{tabular}{|c|c|c|c|c|c|c|c|c|}
\hline \multicolumn{9}{|c|}{ Mechanical Parameters - Estimated Mean \pm Standard Deviation } \\
\hline \multirow{2}{*}{ Zone } & \multicolumn{2}{|c|}{ Neo Hookean Model } & \multicolumn{3}{|c|}{ Ogden Model } & \multicolumn{3}{|c|}{ Holzapfel-Gasser-Ogden Model } \\
\hline & $\mathrm{C}_{10}(\mathrm{MPa})$ & $R^{2}$ & $\mu(\mathrm{MPa})$ & Alpha (-) & $R^{2}$ & k1 (MPa) & k2 (-) & $\mathrm{R}^{2}$ \\
\hline Supraumbilical & $0.61 \pm 0.34$ & $0.73 \pm 0.13$ & $0.39 \pm 0.37$ & $38.57 \pm 15.55$ & $0.99 \pm 0.01$ & $0.46 \pm 0.36$ & $63.03 \pm 38.42$ & $0.99 \pm 0.01$ \\
\hline Infraumbilical & $4.08 \pm 1.94$ & $0.89 \pm 0.06$ & $4.61 \pm 2.54$ & $35.73 \pm 14.79$ & 1.00 & $5.04 \pm 2.67$ & $77.04 \pm 63.91$ & 1.00 \\
\hline
\end{tabular}




\section{FIGURE LEGENDS}

Figure 1. (a) Schematic of the porcine abdominal wall and location of the 30 samples obtained. (b) Picture of two dissected samples and definition of the dimensions HO, WO and t0. (c) Schematic of the tensile test machine and S-DIC system used to measure local displacements and strains.

Figure 2. Geometrical dimensions comparison of linea alba samples.

Figure 3. Confocal microscopy of the collagen fibers in linea alba. (a) infraumbilical sample, outer side. (b) supraumbilical sample, inner side. The black dotted lines represent the fiber directions."

Figure 4. Typical stress-strain response curves of linea alba to planar tension.

Figure 5. Stretch-(fitted) stress curves and standard deviation for supraumbilical and infraumbilical zones.

Figure 6. Comparison of the stretch/stress curves for linea alba. 